\title{
Surge Protective Device Failure Modes, Impact and Corrective Actions
}

\author{
Arati Channe \\ Currently Pursuing Master's Degree \\ in Electrical Engineering Power System in Walchand College \\ of Engineering, Sangli.
}

\author{
Vijay Mohale \\ Currently Working As Assistant Professor \\ in Walchand College of Engineering, Sangli
}

\begin{abstract}
Surge protective device (SPD) is used to protect electrical and electronic devices from the transient over voltages(surges). Failure occur in SPD effect on the expected performance of overall protection system. A more thorough understanding of all possible SPD failure modes is important for system protection. The main motive of this paper is to provide the reader with principles of SPD failure and effective corrective action.
\end{abstract}

Keywords: Corrective action, Failure, Impact, Metal Oxide Varistor (MOV), Surge, Surge Protective Device (SPD), Temporary overvoltage (TOV), Transient.

\section{INTRODUCTION}

The increased use of sensitive electrical and especially electronic devices and system brought about a marked increase in failure rates due to dangerous induced voltages. Surge Protective Device (SPD) used to protect the electrical and electronic equipment from damage by power surges.

When specifying and installing any Surge Protective Device (SPD) in low-voltage power distribution equipment [1] associated with commercial and industrial installations, numerous application considerations should be reviewed and evaluated before installation. Maloperation of SPD can directly impact on the expected performance of the protection system and can result in undesirable effects like damage and burn the component.

\section{SPD Failure History}

In this paper Table 1 showed classification SPD failure over a three-year period, for series integrated SPD. Theleading cause of SPD failure is mal operation.

TABLE 1

DEFECT COUNT OF SPD

\begin{tabular}{|c|c|}
\hline Type of defect & $\begin{array}{l}\text { Count of } \\
\text { Defect }\end{array}$ \\
\hline Maloperation & 75 \\
\hline $\begin{array}{l}\text { Functional } \\
\text { Failure }\end{array}$ & 25 \\
\hline Alarm & 5 \\
\hline powering issue & 2 \\
\hline Tripping issue & 1 \\
\hline
\end{tabular}

To overcome recurring issues of SPD, carried out the root. cause analysis and give corrective action.

\section{TRANSIENT OVERVOLTAGE}

\subsection{Surges}

A short duration increases in voltage measured between two or more conductors is known as transient over voltages (surges). Surge [2] duration is from microseconds (millionths of a second) to a few milliseconds (thousandths of a second). During the transient condition sudden increase in voltage will change from a few volts to thousands of volts. voltage produces between two or more conductors. These conductors would be the line/phase, neutral and earth for a mains power supply.

\subsection{Surge Origins}

Surge origin sources areas follow [1]

1. Lightning surges: In this a direct flash to the power system, to the structure of interest and nearby structures, or to the soil. Lightning surge produce very high potential (voltage) difference between two conductor and ground. Due to distant lightning flashes voltage surges induce in the circuits of an installation.

2. Switching surges: Switching surges are intended actions on the power system, such as load or capacitors switching. These may also be the product of unintentional occurrences such as faults in the power system and subsequent corrective action.

\subsection{Problem Caused by Transient Over Voltage}

The effects of transient overvoltage's, whether produced by lightning or electrical switches, are similar

1. Disruptive damage: An electronic component that interprets the valid logic command is entered. The resulting lockup system, software failure contributing to incorrect results, or missing information.

2. Dissipative: A repetitive, short-lived energy pulsing, long term machine or system degradation resulting in earlier intervals of system replacement.

3. Destructive: A high-level surge of energy that leads to equipment failure or destruction immediately. Although many surges are not automatic, with a validated and checked surge protection system for prevention of damage to a facility. 


\section{SURGE PROTECTIVE DEVICES}

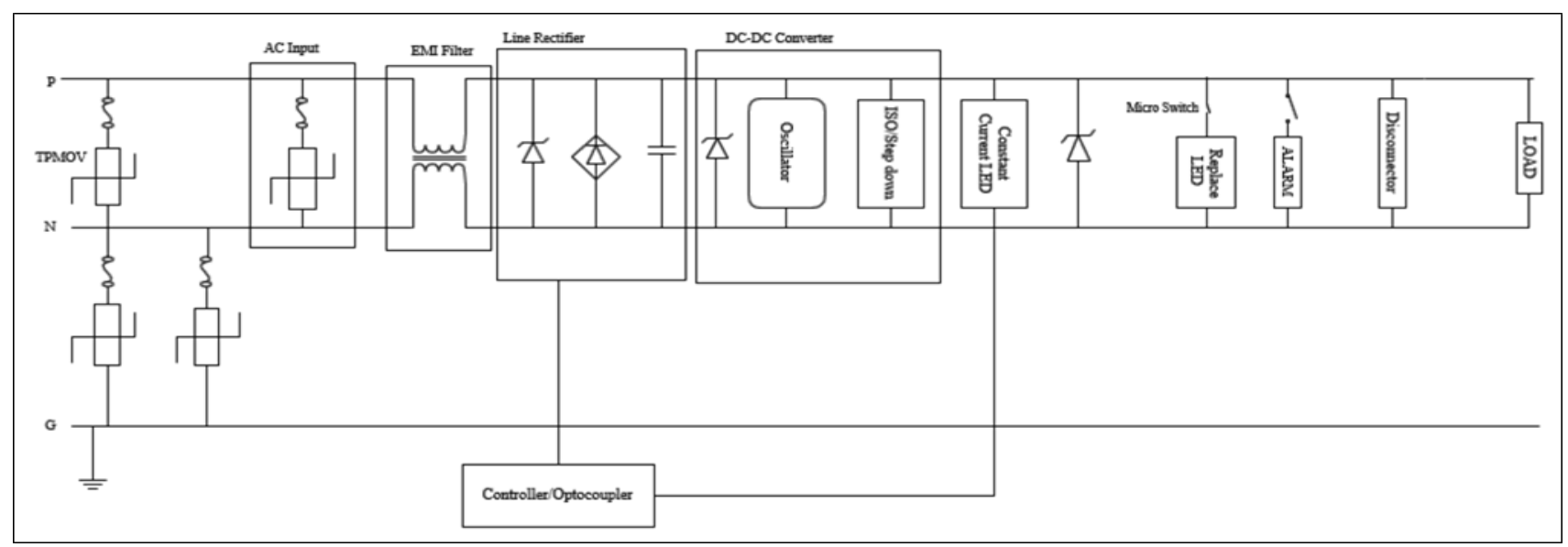

Fig. 1. Internal block diagram of SPD.

Surge protection devices (SPDs) can work in surge conditions. Normally, SPD will do absolutely nothing, much as a fuse does nothing when it is used within its rating and if for example, the voltage in the network increases due to a lightning strike, the SPD starts to drive the energy away from the critical unit. Unlike a fuse, though, it is possible to use a surge protection system many times.

In Fig. 1. as shown Ac line fuse with acceptable $I^{2} T$ rating essential to pass energy star ring wave test as stated by IEEE C.62.41. TPMOV for ac line use to protect LED bulb from transient condition(surges) and ring wave effect by clamping short duration voltage impulses. The high frequency noise may cause interference with other devices to condenses this use Electromagnetic Interference (EMI) filter. Before, line rectifier place TVS diode for components sensitive to and easily damaged by surge voltage or current. TVS diode used before DC-DC converter to keep against transient pass into converter circuit. When the MOV no more in use the micro switch get signal and replaced LED get on. TVS used before replaced LED, transient protection for energy let through to LED circuit. Once this happens, the unit (MOV) will need to be replaced.

\section{THERMALLY PROTECTED METAL OXIDE VARISTOR (TPMOV)}

Metal oxide varistors (MOVs) [3] are frequently used in combination with current limiting fuse elements in SPD. MOVs are two-terminal electrical systems with nonlinear relationship between voltage and power. MOV shows a high impedance between its two terminals at low voltages, but at voltages higher than a fixed peak voltage, the impedance changes rapidly to a small impedance. This characteristic is useful as a voltage limiter. Because as the voltage across the MOV terminals increases, within the power handling ability of the MOV and the voltage is clamped to the predetermined limit voltage. The predetermined limit voltage is a characteristic dependent on a MOV and is determined by the MOV manufacturing process.

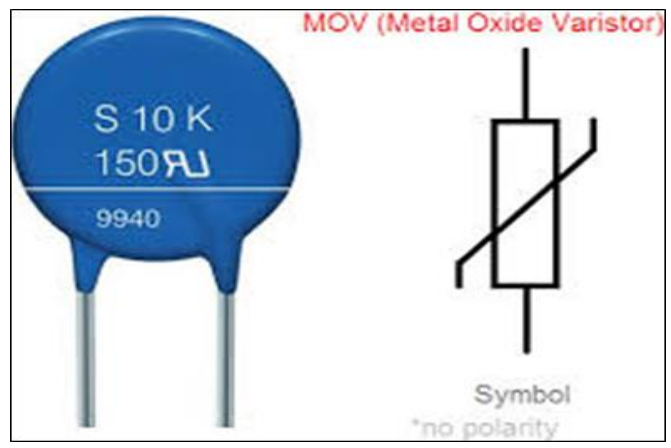

Fig. 2. MOV

A problem with typical MOVs is that under the repeated power surges, a classic varistor will gradually deteriorate [4] until it eventually results in a serious malfunction. Therefore, the MOV need to be replaced before the component deterioration reaches the point of failure. To overcome this problem, develop the new technology called Thermally protected MOV.

TPMOV contains metal oxide varistor (MOV), Heat sensor (Thermal element) direct in contact with varistor surface and spring-loaded shield made up of an insulating material. Voltage flows through two elements. The varistor Current flows through the spring element and through the thermal element to the opposite side of the varistor. This, thermal element is designed to wrap when overheated. When the varistor fails, it overheats to extreme temperature causing smoke and often ignite the causes fire. This failure is where TPMOV technology 
comes into play. Upon Varistor failure, TPMOV technology prevents the varistor from excessive overheating and thus prevents the possibility of dangerous smoke and fire. When the varistor fails and heats up, then thermal elements heat up and wraps, which causes it to release the arc shield. As the thermal element releases the arc shield, the springs push it upward, causing the shield to cover the varistor and prevents any current flow from the thermal element to varistor. This prevents the varistor from continuing to overheat and cause smoke or fire.

\section{FAILURE MODES, IMPACT AND CORRECTIVE ACTIONS}

\subsection{Failure mode of SPD might include the following}

\section{Increase in power follow current or leakage current beyond specifications [2]}

This happen due to sudden increase in load or switch-off the supply. Then produce over voltage, undervoltage in the system. To overcome this use loss of load protection, auto tap changing transformer for voltage control.

\section{Unintended flashover}

Produces due to high voltage electric discharge over or around an insulator and sparking occur between two or more adjacent conductors. This effects on electrical grounding systems (grounded pipe work) and is conducted back through these into the building where it can travel through the electrical system, creating heavy damage along its path. To protect the system from unintended flashover, use the appropriate value of low resistance in grounding which give the path to flashover and yearly/ half yearly check the earth resistance as per preventive maintenance plan.

\section{Degradation}

Occur due to premature internal failure and operation conduction which produce some heating in the SPD. So that changes voltage relief ability of SPD by lowering the conductive threshold level or decreasing the Maximum Continuous Operating Voltage (MCOV) window. By providing the proper enclosure and thermal component help to minimize the degradation problem.

\section{Overheating}

Overheating [2] results in a hazardous condition creates when semiconductor devices or MOV gets damage. Due to this Degrades the SPD performance Compromise SPD life. Hence need to give the proper enclosure and cooling to reduce the overheating.

\section{SPD co-ordination failure}

Generally, occurs due to improper distance between SPDs in the Power Distribution System (PDS), including classification by SPD exposure type, Location Category. Incorrect SPD co-ordination methodologies which directly influence the expected performance of the protection system. So, follow the category definition for the proper distance selection [5-6].

\section{Thermal Runway}

Produces due to overvoltage, Continuous overcurrent flow through circuit and SPD end of life effect on device increase the internal thermal heat, melt the solder wire. If thermal runway [2] remains for long time, then SPD out of services so to overcome the problem

1. The use of an appropriate enclosure.

2. Encasement of the Surge Protective Component (SPC) selections within suitable sealing compound, sand or both. 3. Use of internal or external overcurrent protection or SPD disconnector(s).

4. Use of integrated thermal cutoff components such as fuse connections, soldering tape, thermal squibs or thermal dis connectors. Avoid or limit the use of flammable materials in the enclosure.

5. Any combination of any or all the above.

\section{Failure due to TOV}

1. Loss of neutral (lack of reference to ground).

2. Loss of phase (i.e. lose power to one or two phases of a three-phase system).

3. Ground faults on ungrounded electrical systems misapplication of the SPD (i.e. $208 \mathrm{~V}$ SPD applied to $480 \mathrm{~V}$ ).

4. Transmission line contacting distribution line (comingling).

5. Voltage regulation issues.

6. Earth fault occur on the line side of service apparatus.

7. Earth fault takes place on the load side of service equipment.

8. Power distribution systems subject to drastic load changes.

Due to which Magnitude or duration of temporary overvoltage increase. SPD failure due to thermal stress as the SPD shunts power frequency current during the overvoltage which lasts for seconds to minutes. To avoid this failure the end user should consider an SPD having a higher MCOV or one that is certified to properly mitigate the effects of TOVs (disconnect from or withstand the event).

\section{Serviceability/Accessibility}

Failure are services or maintenance will not be performed i.e. not replaceable modules, batteries, or other components. So, need to do the yearly maintenance.

Residual current device

Fails to operate due to wrong trip setting and improper earthing produce unwanted tripping, to overcome this follow the trip setting and earthing as per guideline.

Shorting or opening of any protective circuitry including a fuse, thermal protective device, or circuit breaker

Cause due to latching problem of circuit breaker, fail to operate the protective device, selection of fuse and protective device of improper rating. Hence, increase in internal overcurrent have chance of burn and damage internal component of SPD. Corrective action on it is check the latching of C.B and tripping setting. Use fuse, thermal protective device of proper rating.

5.2 Failure Mode Due to installation Improper installation includes [2]

1. Improper grounding and bonding method

2. Open transition switch

3. Open neutral connection

4. Phase loss conditions

5. TOV of unloaded transformer

6. Improper MCOV selection

7. WE-configured SPD installed into a delta system

8. Screw tightening torque

9. Loose connection 
10. Distance between SPD and panel board connected

11. Wrong wire connection

Due to which have chance to go SPD into conduction and malfunction. Therefore, follow the proper installation process as per given guideline to obtain the desired operation.

\section{Contact failure}

Loose connection lead to melt wire due to more heat Soldering issue produce the sparking and more losses takes place. In severe condition cause open or short circuit. After the installation check the connection, take insulation resistance test and use appropriate the solder paste for soldering [8].

\subsection{Failure due to component LED failure}

Material, structure, packaging technique, using condition are the factors which cause LED fail [7]. Failure modes of LED are

1. Chip failure: Defect in chip or another source make chip failed.

2. Packing failure: Inappropriate packing design and manufacture procedure.

3. Failure due to thermal stress: Produced by LED internal temperature reach to permissible value or cyclic heat varying in LED.

4. Electric stress: Produce due to exceeded rated electrical parameter or high transient current.

5. Assembling failure: Improper method of assembly during the actual process.

\section{Method to improve LED reliability}

\section{Thermal Management}

In internal thermal management thermal resistance handled from junction to the package case completed during design process and plays an important role. External thermal control involves cooling mode selection, heat sink configuration, material selection and installation process.

\section{Optimize packaging cycle}

Bonding Cycle optimum condition regulated by form of cable, configuration of the pad metallizing system. Die attachment procedure Chip pad testing should be clean and free of dust. Chip should be complete without frame breakage, should not be rusty and deformation silver filled die connection content should not be overdue.

\section{Screening technology [7]}

Monitoring equipment uses a reasonable range of ageing and monitoring approaches such as temperature cycling, high temperature preservation and enough study burning to prevent infant loss.

Printed circuit board (PCB) failure

\section{Component Layout Failure}

Improper unit positioning, poor part positioning, lack of space on the PCB and system barrier breaking arises power failure and overheating. To overwhelm this, increase thermal pad isolation, balance number connections for component per lead each plated through hole uniformity of trace size connection to component plated through hole.

\section{Poor Quality Components}

In this include bad soldering to cold joints, poor connectivity between circuit boards, insufficient board thickness use of counterfeit components. Effect on material i.e. bending and breaking. To mitigate it use the proper thickness of board as per rating and required Solder paste [8].

\section{Environmental Factor}

This includes dust, moisture, power overloads/surges, premature circuit board failure due to electrostatic discharge. So that exerts the thermal stress on PCB. Remedy for this problem, need the cleaning and maintenance of PCB, Proper neutral grounding.

The opening or shorting of electromagnetic interference circuitry (Resistor, inductor capacitor) [2]

This scenario creates due to excess temperature, excess current or voltage ionization radiation mechanical shock. Therefore, it melts the soldering wire, burn the component, interference problem produce smoke. For better utilization of SPD, use the resistor, inductor and capacitor of proper rating. Follow strict maintenance to know any module come to replace.

Metal oxide varistor (MOV) [4]

MOV fail due to Short Circuit degradation age, this happens because of thermal runaway, environmental degradation, material defect, insulation degradation, degraded connections, defective circuit. Formulation of Smoke and fire increased in leakage current towards ground. In such condition must replace MOV.

Sometime MOV has damage like Crack, epoxy breakaway, puncture. So, need to check before the mounting of MOV on PCB to avoid thermal runway problem.

\section{Optical Isolators}

Optical isolator fails due to degradation which rises due to age, irradiation hardening, environmental degradation, insulation degradation, degraded connections, defective circuit. Decrease in LED brightness. Corrective actions for it provide proper thermal management and screening technology.

\section{Common Mode Filters}

Factor which are responsible for failure are age, irradiation hardening, environmental degradation, insulation degradation, degraded connections, defective circuit. Failure of filter produce dielectric breakdown in capacitors, insulation breakdown and inductor arcing. By using the high resistance insulation, proper maintenance also helps to know any module come to replace or not.

5.4 Failure mode due to environment condition [2] Factor which are responsible for the failure of SPD might include fatigue, environmental gases (water vapor), moisture, exposure of energized equipment to abnormal vibrations or shocks, excessive vibration. Due to this produce effect swelling of IC package, electrical shortening corrosion of metal component. In environmental ratings include enclosure rating, operating temperature range, storage temperature which need to consider during the design of SPD.

\section{CONCLUSION}

SPD is one of the protective equipment which is used against the transient over voltage. Transient over voltage (Surges) can damage the equipment and other electrical component in electrical distribution. It is essential, to prevent power system from power surges. But when failure occur in SPD effect on 
protection system and cause nuisance equipment tripping. Therefore, it is necessary to avoid malfunctioning of SPD.

Overall work in this paper, impact of failure mode, origin of causes and suggested corrective action to enhance the capability and performance of SPD.

\section{REFERENCES}

[1] Dev Paul, Senior Member, IEEE, and Srinivasa I. Venugopalan, Senior Member, IEEE "Power Distribution System Equipment Overvoltage Protection" IEEE transactions on industry applications, vol. 30, no. 5, september / october 1994.

[2] Surge Protective Devices Committee of the IEEE Power and Energy Society "Draft Guide for the Application of SurgeProtective Devices for Use on the Load Side of the Service Equipment in Low Voltage $(1000 \mathrm{~V}$ or Less, 50 or $60 \mathrm{~Hz}) \mathrm{AC}$ Power Circuits" 2015 by The Institute of Electrical and Electronics Engineers, Inc.

[3] Naoyuki Tsukamoto, Member, IEEE, and Masaru Ishii, Fellow, IEEE "Repetitive Impulse Withstand Performance of Metal-Oxide Varistors" IEEE transactions on power delivery, vol. 32, no. 4, august 2017 .

[4] S. Ioannou Student Member IEEE, E. K. Stefanakos Senior Member IEEE and P. H. Wiley Member IEEE "New MOV Failure
Mode Identification Invention" IEEE Transactions on Consumer Electronics, vol. 53, no. 3, august 2007.

[5] G. L. Amicucci, F. Fiamingo, Z. Flisowski, G.B. Lo Piparo, C. Mazzetti, Member, IEEE "Surge protective devices for low voltage systems: practical approach for the protection distance evaluation" 2007 IEEE.

[6] Jinliang He, Fellow, IEEE, Zhiyong Yuan, Shunchao Wang, Jun $\mathrm{Hu}$, Shuiming Chen, Senior Member, IEEE, and Rong Zeng, Senior Member, IEEE "Effective Protection Distances of LowVoltage SPD. With Different Voltage Protection Levels" IEEE transactions on power delivery, vol. 25, no. 1, january 2010.

[7] Guoguang LU, Shaohua YANG, Yun HUANG Key Lab for Reliability Physics and Application Technology of Electrical Component CEPREI Guangzhou, China "Analysis of Failure Modes and Mechanisms of LED" 2009 IEEE

[8] Yaojun Chen, Zengjin Sheng, Bo Jing, Jiaxing Hu "Failure Mode Analysis of Board-Level Solder Joint under Random Vibration Load" 2018 International Conference on Electronics Technology.

[9] G.B. Lo Piparo, T. Kisielewicz, C. Mazzetti, R. Pomponi: Assessment of probability of damage of an apparatus protected by a surge protective devices system, Electrical Power and Energy Systems, ISSN: 0142- 0615, Vol. 75, February 2016. 